\title{
Michael D. Randall and Karen E. Neil: disease management: a guide to clinical pharmacology
}

\author{
3rd Edition, Pharmaceutical Press London, 2016, ISBN: 978085711209 5. Price around $€ 70.00$
}

\author{
J.W. F. van Mil ${ }^{1}$
}

Received: 4 October 2018 / Accepted: 8 October 2018 / Published online: 10 October 2018

(c) Springer Nature Switzerland AG 2018

Clinical pharmacology is a topic that is essential for clinical pharmacists. If you select, apply and review medicines, and monitor the patients' wellbeing and quality of life, you must know about the treatment of their diseases. Not every (student) pharmacist learns about the optimal treatment of diseases, but if you want to practice in a setting together with medical doctors you should be able to speak their language, and share their approach to health and disease. Clinical pharmacology is typically the field of expertise for this interface between the professions. This book can be very helpful in increasing the pharmacist knowledge about the use of medicines in specific diseases, and thus supporting decisions about patient care.

After some more general (but interesting) chapters on signs and symptoms, clinical laboratory tests, lifestyle and herbal medicines, the second part of the book is dedicated to treatments, drug choices, adverse events and pharmacokinetics. The remaining nine sections are dedicated to specific diseases and their treatments, organized under main groups of diseases: cardiovascular, respiratory, central nervous system, pain, cancer, infections, dermal problems and endocrine disorders. Each chapter generally describes the disease characteristics, pathology, clinical features and treatment goals.

In spite of the very interesting content, there seem also to be shortcomings and structural challenges. I am for instance not sure if the title of the book represents its content well. Disease management indicates more than managing medication only. It relates to general standards and protocols for treating diseases, the roles of other healthcare professionals, and as such certain aspects (non-pharmacological treatment, deprescribing) are not covered in full. The title 'Disease Management' might be a bit misleading. In the second part of the book, I would have welcomed a general chapter and the role of treatment protocols and electronic prescribing.

The choice of disease topics seems somewhat unstructured and perhaps incomplete. Why only diabetes and thyroid diseases in the section endocrine disorders, but no growth hormone deficiency? Why only a general chapter on cancer and chemotherapy, and cancer and palliative care, but no information on the most frequently occurring cancers such as lung cancer, breast, ovarian cancer, and bowel cancer(s), that each have different treatments, and different treatment sequences and duration? Why are the viral infections (hepatitis, AIDS) missing in the section on infectious diseases. Why is 'the renal patient' (if there exists such a patient) put in the chapter of cardiovascular diseases?

While the content of the book is definitely highly interesting for pharmacists and pharmacy students, the organization of topics and chapter could be improved, as well as its comprehensiveness. This book on Disease Management is more a reading and study book, but not so much a reference book. It would be nice if such functions could be combined, and if the book could develop into a more comprehensive work on clinical pharmacology. This book, that already appeared in 2016, might now also need some updates.

J. W. F. van Mil

jwfvmil@vanmilconsultancy.nl

1 Van Mil Consultancy, Margrietlaan 1, 9471 CT Zuidlaren, The Netherlands 